\title{
Postcolonial, gender und science studies als Herausforderung der Soziologie
}

\author{
Von Julia Reuter/Matthias Wieser
}

Zusammenfassung: Postcolonial, gender und science studies wurden aufgrund ihrer unterschiedlichen Phänomenologie häufig als Spezialdiskurse in separaten Bindestrich-Soziologien behandelt. Dabei ist ihre Heuristik ähnlich: Sie problematisieren die Unterscheidung von Natur und Kultur, sie dezentrieren das >klassische $<$ Subjektmodell und kritisieren das > westliche < Projekt der Moderne. In dem Beitrag werden diese Konvergenzen zwischen postcolonial, gender und science studies anhand zentraler Konzepte systematisch herausgearbeitet, um daran anschließend zu fragen, auf welche >blinden Flecken $<$ und Probleme sie die Soziologie aufmerksam machen und wo die Grenzen ihrer Erneuerung der Sozialtheorie liegen.

In der gegenwärtigen sozial- und kulturwissenschaftlichen Diskussion, allen voran in den postcolonial, gender und science studies, haben sich Grenzgestalten und Mischwesen und ihre theoretische Reflexion als transkulturell, queer oder hybrid als fester Bestandteil etablieren können. Dank Edward Said, Stuart Hall und Homi Bhabba wissen wir von der Ambivalenz kultureller Identitätserfahrungen von MigrantInnen. Aber auch vertraute Alltagsphänomene verlieren im Blick derzeitiger Diskussionen zunehmend ihre Eindeutigkeit: So haben Judith Butler und Donna Haraway mit ihren Arbeiten zur Sexualität und Körperlichkeit immer wieder die natürliche Zweigeschlechtlichkeit in Frage gestellt, während Bruno Latour, Michel Callon und John Law uns sogar die Belebtheit der Dinge vor Augen führen.

Trotz ihrer ungleichen Untersuchungs >objekte<, lassen sich alle drei studies in Anlehnung an Urs Stäheli (2000) als »poststrukturalistische« Soziologien verstehen, ${ }^{1}$ die in einer breit angelegten Kritik eines modernen Wissenschaftsprogramms und seinen gesellschaftstheoretischen wie forschungspragmatischen Prämissen konvergieren: Sie thematisieren und hinterfragen die Grenze zwischen ehemals binär und dualistisch gedachten Konzepten, sei es die Grenze zwischen Kulturen, zwischen Geschlechtern und/oder Wissenschaften. Dabei thematisieren sie besonders die brüchigen Figuren der Gegenwart mit dem Ziel, das >klassische< Subjektmodell zu dezentrieren und seine Performanzen aufzuzeigen. Obwohl sie sich jeweils an konkreten Begriffen und Konzepten abarbeiten, sind ihre Analysen in eine umfassende Kritik am >westlichen< Projekt der Moderne eingebettet. Damit bieten sie nicht nur einen neuen Blick auf spezifische Phänomene einer durch und durch globalisierten und technisierten sozialen Welt, sondern eröffnen auch neue sozialtheoretische und wissenschaftspolitische Perspektiven auf die heutige (Forschungs-)Gesellschaft. Die Phänomene sind so letztlich auch als Rückseite einer neuen Gesellschaft zu betrachten, die sich - wie unsere Systematisierung im ersten Kapitel zeigt - in der Rekonfiguration grundlegender Konzepte wie Identität, Differenz und Performanz niederschlagen.

1) In Anlehnung an Serres (1987) definiert Stäheli postrukturalistische Soziologien als Parasiten des wissenschaftlichen mainstreams, die aus dem >Archiv< der neueren französischen Philosophie (Foucault, Deleuze, Derrida, Lacan und Serres) - sprich dem Poststrukturalismus - schöpfen. Es gibt auch von anderer Seite Bemühungen, die unterschiedlichen Ansätze unter einem gemeinsamen Label zu fassen - jeweils mit unterschiedlicher Schwerpunktsetzung: etwa postmodern, postfoundationalist, (french) theory, praxistheoretisch sowie diskurstheoretisch, oder neuerdings auch als Kulturtheorien der >Transdifferenz< (vgl. z.B. Angermüller 2004, Keller 2004, Reckwitz 2003, Lösch 2005; AllolioNäcke/Kalscheuer/Manzeschke 2005).

Soziale Welt 57 (2006), S. $177-191$ 
Während diese Konvergenzen zwischen postcolonial, gender und science studies in der angloamerikanischen Rezeption größtenteils erkannt wurden und unter dem label cultural studies eine kritische Rekonstruktion der bisherigen wissenschaftlichen Paradigmen einschließlich dem Umschreiben der Sozialtheorien angestoßen haben, erfolgte ihre Rezeption in den deutschen Sozialwissenschaften erst sehr spät und meist im Diskussionszirkel spezieller Soziologien. ${ }^{2}$ Hier ist die Beschäftigung mit Grenzkultur- und Randphänomenen immer noch selbst eine Art Grenz- oder Randkulturforschung: Ihre Diskussion bleibt meist im engen Rahmen von theoriepolitisch wie institutionell voneinander getrennten Teil- oder Bindestrichsoziologien (Kultursoziologie, Geschlechtersoziologie oder Wissenschafts- und Techniksoziologie), so dass das Potential und die Herausforderung für die Sozialtheorie häufig übersehen wird. ${ }^{3}$

Dieser Herausforderung wollen wir uns im zweiten Teil dieses Beitrags stellen, indem wir auf die von den studies implizierten sozialtheoretischen Erweiterungen und Revisionen ebenso wie auf >blinde Flecken < und Probleme der Soziologie schauen. Neben dieser Erörterung des Potentials poststrukturalistischer Ansätze für die Soziologie, sollen abschließend aber auch umgekehrt die Grenzen ihrer Konzepte aus soziologischer Perspektive in den Blick genommen werden. ${ }^{4}$

\section{Zwischenräume und Grenzgänger: hybrid, queer, trans}

Auch wenn postcolonial, gender und science studies sehr unterschiedliche Phänomene untersuchen - Kultur-, Geschlechts- und Objektidentitäten -, ist ihre Heuristik ähnlich. Sie irritieren den etablierten Blick auf die Dinge, indem sie die vertraute binäre Einteilung der Welt in eigene und fremde Kultur (postcolonial studies), in männlich und weiblich (gender studies), oder auch Subjekt und Objekt (science studies) hinterfragen. Empirisch wird dies durch den Rückgriff auf Phänomene deutlich, die sich einer einfachen Klassifikation entziehen: Die prototypischen Figuren postkolonialer Studien sind die vielen tausend Migranten, wie sie v.a. in den Metropolen des >Westens $<$ zu finden sind: weder voll assimiliert noch total ausgegrenzt, mit unterschiedlichen kulturellen $>$ Wurzeln $<$, die diese ebenso abstreifen wie pflegen. Die Untersuchungssubjekte der gender studies sind jene, die nicht in die kategoriale Differenz von männlich/weiblich der »heterosexuellen Norm« (Butler 1991) passen: Transgender, Transvestiten, Transsexuelle und Intersexuelle. Science studies beschäftigen sich mit den allgegenwärtigen Mischwesen aus Natürlichem und Kulturellem der technisierten Gesellschaft: Ozonloch, OncoMouse, aber auch Türschließer und Schlüsselanhänger (vgl. Latour 1996a; 1997; Haraway 1997). ${ }^{5}$ Oder allgemeiner formuliert: Im Mittelpunkt der studies stehen Menschen ohne Wurzeln mit brüchigen Identitäten und mehrfachen Zugehörigkeiten, sowie Technologien oder Artefakte, die von Wissen und Ideen durchdrungen sind und selbst wiederum das Denken und Handeln der Menschen durchdringen. So sehr sich die Untersuchungs>objekte< der studies in phänomenologischer Hinsicht unterscheiden, so lassen sie

2) Hier macht v.a. der noch junge Bielefelder Verlag transcript die löbliche Ausnahme, indem er - neben seinem >schicken< Design - gerade durch die Publikation dieser und anderer studies auf sich aufmerksam macht - letzteres etwa in Form eigener (Programm-)Reihen für cultural, gender und science studies. Vgl. für den hier diskutierten Rahmen etwa ganz aktuell Castro Varela/Dhawan (2005), Hieber/Villa (2006) und Belliger/Krieger (2006).

3) Vgl. hier etwa englischsprachige Fachlexika wie z.B. Elliott/Turner (2001) und Lemert (1999), in denen auch Einträge zu Donna Haraway, Stuart Hall und Edward Said bzw. zu Frantz Fanon, Laclau/ Mouffe und Judith Butler zu finden sind, welche man in deutschen Fachlexika und Lehrbüchern der Soziologie vergeblich sucht. Vgl. hierzu auch Reckwitz (2002).

4) Ganz ähnlich hat kürzlich Sérgio Costa (2005) die von postkolonialen Theorien an die Soziologie gerichtete Kritik untersucht und ihre Herausforderungen für eine Revision modernisierungstheoretischer Makrosoziologien entworfen. 
sich doch als »epistemologische Geschwister« begreifen (Nassehi 1999: 356): Sie entstellen allesamt die binäre Logik, mit der auf Differenz beruhende Identitäten für gewöhnlich konstruiert werden. ${ }^{6}$ MigrantInnen mit ihren unterschiedlichen kulturellen $>$ Wurzeln $<$ irritieren die Nationalkultur und ihre Identitätspolitik des >ethnischen Absolutismus $<$. Trans- und Intersexuelle unterlaufen die Vorstellung vom > wahren< (Zwei-)Geschlecht. Sozio-technische Netzwerke erschüttern die Definition von Subjekt/Objekt und offenbaren die kulturelle Dimension von Objekten und (Arte-)Fakten und die materielle Dimension von Kultur. ${ }^{7}$

Theoretisch findet diese (Wieder-)Entdeckung der Phänomenologie des >DazwischenSeins in Begriffen des Hybriden (Bhabha 2000; Latour 2000; 2002; Schneider 2000), des Transkulturellen (Welsch 1997) oder auch in dem taktischen Wiedereinsatz des Queer-Begriffs (Butler 1991; 1995) seinen Ausdruck, die in jenen Ansätzen eine Schlüsselposition einnehmen. Dabei stehen die neuen Begriffe nicht für eine bloße Kombination von ehemals >harten< Entitäten, sondern für prozesshafte, dynamische, flüssige Vermischungen, die sich nicht ohne weiteres feststellen lassen, weshalb Susan Stanford Friedman auch von einem »zwielichtige[n] Übergangsstadium des >Dazwischenseins « s spricht (Friedman 2003: 35).

Postcolonial, gender und science studies betonen die wechselseitige Bedingtheit ehemals kategorisch gedachter $>$ Einheiten $<$. Insofern lassen sie sich auch als anti-essentialistische Prozesstheorien verstehen, die sich von verdinglichenden Tendenzen eines binären Differenzdenkens deutlich abgrenzen: So kritisieren bspw. postkoloniale Arbeiten die exklusive Gegenüberstellung von eigener und fremder Kultur in der klassischen Soziologie des Fremden und betonen stattdessen ihre wechselseitige Bedingtheit und Relativität (vgl. Ha 1999; Reuter 2002). Und auch neueren Arbeiten der gender studies haben eine Debatte zur lange Zeit dominanten sex/gender-Unterscheidung angestoßen. Sie betonen, dass sich biologische und kulturelle Geschlechtsidentität nicht mehr voneinander trennen und Frauen und Männer immer weniger als homogene Gruppe gegenüber stellen lassen. Ihr kritischer Impetus in Bezug auf bestehende wissenschaftlichen Traditionen und Ansätze ist ähnlich: Analog zum Vorwurf der postcolonials gegenüber dem Panafrikanismus, der black panther-Bewegung oder den subaltern studies, den Binarismus aufrechtzuerhalten und die Differenz durch Umkehrung der Hierarchie lediglich zu substantialisieren (vgl. Hall 1994: 18, 20; Spivak 1988), kritisiert

5) Auch das persönliche wie professionelle Selbstverständnis der AutorInnen ist vom Motiv des >grenzgängerischen Hybriden > gekennzeichnet. Bruno Latour (1996a) etwa sieht sich als Grenzgänger zwischen den Disziplinen: Er sei Philosoph, Soziologe, Ethnologe, Semiotiker und »Liebhaber der Wissenschaften « in einer Person. Auch der kürzlich verstorbene Literaturwissenschaftlicher Edward Said hat sich Zeit seines Lebens als Grenzgänger verstanden. Die Gegensätzlichkeiten, die das Leben dieser Intellektuellen prägt - Said, sowohl amerikanischer Araber als auch palästinensischer Protestant, sowohl heimatloser Vertriebener als auch New Yorker - ist dabei Programm; zumindest erscheint die persönliche Hybridität als Inspirationsquelle wie Autorisierung ihrer theoretischen Reflexion von Grenzlagen.

6) Sie befinden sich in einem ambivalenten Zwischenstatus und können nach Zygmunt Bauman (1995: 73ff.) als Fremde bezeichnet werden, die sich que(e)r zu binären Ordnungs- und Vergesellschaftungsformen stellen. Als Prototypen des vertrauten Fremden sind sie vertraut wie fremd zugleich (vgl. Nassehi 1995), denn sie liegen einerseits jenseits der vertrauten internen Unterscheidungen wie einheimisch/ausländisch, Mann/Frau bzw. zwischen Kultur/Natur. Sie machen die Unterscheidungen aber andererseits ihrerseits reflexiv, gewissermaßen als ihr eingeschlossenes-ausgeschlossenes Drittes.

7) Natürlich bilden MigrantInnen, Transsexuelle und Objektidentitäten/Cyborgs nicht die gesamte Phänomenologie der studies ab, noch weniger steht ihre Repräsentanz für eine statistische Repräsentanz des Personals gegenwärtiger Gesellschaften. Aber sie besitzen als prototypische Phänomene für die Analyse der studies gewissermaßen Modellcharakter. In Anlehnung an Max Webers idealtypische Methodologie lassen sie sich auch als >reine< Typen verstehen. 
auch Butler (1991: 21-24) am klassischen Feminismus, dass dieser die Geschlechterasymmetrie aufrecht erhält und die Ausbeutungs- und Unterdrückungsstrategien lediglich umkehrt:

...das Insistieren auf der Kohärenz und Einheit der Kategorie >Frauen $<$ hat praktisch die Vielfalt der kulturellen und gesellschaftlichen Überschneidungen ausgeblendet, in denen die mannigfaltigen konkreten Reihen von >Frauen< konstruiert werden. (Butler 1991: 34)

Auch innerhalb der science studies geht es darum, alte Unterscheidungen in der Tradition >westlicher<Wissenschaft aufzubrechen, etwa zwischen Natur und Kultur sowie zwischen Technik und Gesellschaft, und ihre Verflechtungen herauszustellen. Dies wird sowohl von Seiten einer techniksoziologischen wie feministischen Wissenschaftstheorie forciert: So wie Bruno Latours und Michel Callons Actor-Network Theory für die Symmetrisierung von menschlichen und nicht-menschlichen Entitäten eintreten (vgl. z.B. Latour 2002, Callon 1986), thematisiert Donna Haraway (1995) die Entgrenzung von Mensch und Tier, Organismus und Maschine und Physikalischem und Nichtphysikalischem in den Technowissenschaften des 21. Jahrhunderts. ${ }^{8}$ Latour wie Haraway lehnen jeglichen Dualismus von Maschine und Mensch, Technik und Gesellschaft, Natur und Kultur ab und stellen stattdessen das Programm einer »Symmetrischen Anthropologie« (Latour 2002) oder auch einen »Cyborg-Mythos « des »cat's cradle (Haraway 1995) entgegen. Die Grundannahme ist dabei identisch: Keine Dichotomie liegt a priori fest, sondern ist ein in der Praxis umkämpfter Prozess zwischen verschiedenen, auch nicht-menschlichen, Akteuren. ${ }^{9}$

Aufgrund ihrer Problematisierungen binärer Differenzen und ebensolcher Argumentationsund Analysemuster können postcolonial, gender und science studies auch als Theorien der $>$ Transdifferenz< bezeichnet werden. Schließlich ist das Herzstück transdifferenten Denkens die Unzulänglichkeit binärer Differenzvorstellungen und -konstruktionen als (allgemeingültigen) Erklärungsansatz (vgl. Kalscheuer 2005), sowie die Betonung von Bewegung, Kontingenz und Macht vor dem Hintergrund eines praxeologischen Kulturverständnisses. Letzteres zeigt sich etwa darin, dass sich die Idee der Grenzüberschreitung vor dem Hintergrund einer Absage an die Vorstellung homogener, geschlossener Kulturen und zugunsten der eines Doing Culture (Reuter/Hörning 2004) entfaltet. Allerdings predigen >transdifferente< Ansätze keinen Kulturalismus oder radikalen Kulturrelativismus. In unterschiedlichen Schwerpunktsetzungen kritisieren sie radikalkonstruktivistische Ansätze und heben immer wieder auch die materielle Dimension des Praktizierens von Kultur hervor (vgl. Wieser 2004). ${ }^{10}$

Mit dem Konzept der >Transdifferenz< wird gewissermaßen seitenverkehrt argumentiert: Wo Ordnung weniger in stabilen Strukturen als vielmehr in der prozessualen Handlungspraxis der Akteure liegt, kann es auch keine stabile Differenz geben (vgl. Kalscheuer 2005). Dennoch ist in Anlehnung an Klaus Lösch (2005: 27) der Begriff der Transdifferenz »[...] nicht als Überwindung von Differenz, als Entdifferenzierung oder höhere Synthese misszuverstehen, sondern bezeichnet Situationen, in denen die überkommenen Differenzkonstruktionen auf der Basis einer binären Ordnungslogik gleichsam ins Schwimmen geraten und in

8) Mit Technowissenschaften versucht Haraway (1997) eben jene Vermischungen von Wirtschaft, Wissenschaft und Technik zu erfassen, die für zeitgenössische (v.a. Natur-)Wissenschaften wie die Molekularbiologie und Gentechnik typisch sind.

9) Für eine kritische Auseinandersetzung mit Haraway und Latour, die v.a. auf ihre Differenzen abstellt, vgl. Weber (2003). Neben den hier Angesprochenen gibt es noch weitere poststrukturalistisch informierte oder zumindest inspirierte Ansätze innerhalb der science studies wie etwa Knorr-Cetina (1997) oder Pickering (1993).

10) Hier liegt auch der größte theoretische Knackpunkt unserer > vereinheitlichenden < Argumentation. Während bei den gender studies und den postcolonial studies die Berührungsängste zu Etiketten wie konstruktivistisch, postmodern oder sogar relativistisch recht gering ist, grenzen sich die science studies hier sehr scharf ab, was natürlich auch im Phänomenbereich (Artefakte!) begründet liegt. 
ihrer Gültigkeit temporär suspendiert werden, ohne dass sie damit endgültig dekonstruiert würden.« Transdifferentes Denken will also Differenz nicht auf einer höheren Ebene etwa jene der Hybridität auflösen. Differenz bleibt bestehen, wird allerdings temporär außer Kraft gesetzt, so dass jene ambivalenten Grenzorte entstehen, von denen Friedmann (2003) spricht, oder in Löschs Worten zeigt dies »Zonen der Unbestimmtheit..., die sich einer klaren und eindeutigen Zuordnung sperren « $(2005: 33 f){ }^{11}$

In diesem Sinne erscheint der Begriff der >Transdifferenz $<$ gegenüber anderen Begriffen, wie etwa Hybridität oder queer, im Vorteil: Er intendiert nur eine Relativierung, nicht radikale Dekonstrukion der Differenz, er verbindet unterschiedliche Erfahrungsebenen und ist in seiner Phänomenologie und Theorie prinzipiell >offen< (Kalscheuer 2005). Dieser prinzipiellen Offenheit des Transdifferenzbegriffs steht die >freiwillige wie unfreiwillige < Historizität und Taxonomie von Hybridität und queer gegenüber: Aufgrund seiner problematischen biologischen Herkunft verleitet der Begriff Hybridität dazu, (a) Differenzen zu homogenisieren (und verabsolutieren) und (b) auf einer höheren Ebene aufzulösen. Und auch wenn queer jenes »quer hindurch « (Lösch 2005: 27) des >trans<-Aspektes betont, ist er mehr noch als der Begriff der Hybridität auf einen bestimmten Kontext beschränkt ${ }^{12}$ : auf den der gay and lesbian studies wie auch der Trans- und Intersexuellenforschung.

\section{Identität, Differenz und Performanz}

Die Gemeinsamkeiten poststrukturalistischer Soziologien lassen sich aber nicht unter theoriesystematischen Gesichtspunkten mit Hilfe analytischer Konzepte wie >Queer $<$, der >Hybridität< oder >Transdifferenz < fassen. Auch auf der Gegenstandsebene der studies finden sich im Hinblick auf die Thematisierung und Reflexion von Identität, Differenz und Performanz zahlreiche Parallelen. Aus argumentationsökonomischen Gründen und zur besseren >ÜberSicht gehen wir bei unserem Systematisierungsversuch schematisch vor, indem wir uns einerseits auf jeweils eine/n prominente/n VertreterIn - Homi Bhabba, Judith Butler, Bruno Latour - und auf die Gemeinsamkeiten der studies beschränken. ${ }^{13}$

Grundsätzlich stellen postcolonial, gender und science studies klassische, d.h. cartesianische zentrierte Subjekt- und Identitätstheorien in Frage bzw. dekonstruieren diese. Identität wird als unendliche Produktion und Positionierung verstanden - zwischen Kulturen, Geschlechtern und auch menschlichen und nicht-menschlichen Aktanten. So stellen sie der cartesianischen Identität unstabile, flüssige, werdende Identitäten gegenüber, ohne sie jedoch gleichzeitig >individualistisch zu fassen. Denn obwohl sie die kulturelle Identität, die Geschlechtsidentität und auch die Identität eines Aktanten als produzierte und generierte Phänomene ansehen, sind es nicht rationale, reflexive Akteure, die diese Identitäten konstruieren oder gar basteln. Identität bleibt Verhandlungssache, ein »Kampf um Bedeutungen« innerhalb von Diskursen, Machtoperationen und/oder Netzen. Gerade in diesem Punkt zeigen sich die poststrukturalistischen Wurzeln der drei studies, denn das Subjekt ist Effekt der es konstituierenden Strukturen, jedoch nicht, wie im Strukturalismus, durch diese determiniert - den studies geht es gerade darum, eine andere Position in die klassische Handlung/Struktur-Diskussion einzubringen: Handlung ist weder determiniert noch determinierend.

11) Allerdings, und hier besteht vielleicht ein Unterschied, haben einige AutorInnen wie z.B. Bhabha und Haraway die Hoffnung, in Zukunft diese Differenzen aufzulösen.

12) Lösch (2005: 44) sieht den Begriff Hybridität ausschließlich im postkolonialen Kontext. Allerdings lassen sich auch die science studies als Studien der >Transdifferenz< verstehen und gerade in deren Kontext wird auch der Begriff der Hybridität benutzt (vgl. Latour 2002).

13) Natürlich sind wir uns bewusst, dass eine solche Metaperspektive in synthetischer Absicht den Feldern als Ganzes nicht gerecht werden kann und sogleich als Differenzgenerator fungiert. 
Darüber hinaus (an)erkennen sie die Hinfälligkeit der Unterscheidung von Natur und Kultur: So gilt der Ausspruch Stuart Halls »Weder das >weiße< oder >schwarze< Subjekt wird über die Natur stabilisiert« (vgl. Hall 1994: 21), eben auch im Kontext der gender studies für das männliche oder weibliche Subjekt oder aber im Kontext der science studies für den menschlichen und nicht-menschlichen Aktanten. Alle drei weisen einen > wahren Kern<bzw. $>$ Ursprungsmythos< von Identität zurück, indem sie darauf verweisen, »daß es keinen >Täter hinter der Tat gibt<, sondern daß der Täter in unbeabsichtigter, veränderlicher Form erst in und durch die Tat hervorgebracht wird «(Butler 1991: 209).

Damit stellen sie die Zentralität von Performanz für die Herstellung von Identitäten in den Vordergrund. Mehr noch: Sie fassen Identität selbst als Performanz, Übersetzung und Vermittlung auf, denn so wie eine Übersetzung von einer Sprache in eine andere nie eine 1:1 Übertragung ist, ist auch die kulturelle Identität, Geschlechtsidentität und die Identität eines Aktanten das Ergebnis differenzproduzierender Wiederholungen. Während postkoloniale Arbeiten dies vor allem durch das Aufeinanderstoßen unterschiedlicher kultureller Wissensbestände im alltäglichen Handeln von und mit MigrantInnen verdeutlichen, sprechen die VertreterInnen der science studies von einer Kreolisierung von Wissens- und Handlungsrepertoires durch die permanente Konfrontation menschlicher und nicht-menschlichen Agenten. Hier finden »Vermittlungen« statt, in der beide modifiziert werden und etwas Neues entsteht. Besondere Akzentuierung erfährt der Begriff der »performativen Identität« jedoch innerhalb der gender studies. Judith Butler verwendet den Begriff explizit, um deutlich zu machen, dass das Subjekt und seine (Geschlechts-)Identität ein Effekt - nicht Ursache - performativer Akte ist, wie bspw. Sprechakte (Äußerungen, Zitate) oder identifikatorische Praktiken (vgl. Reuter 2004). Oder anders formuliert: Geschlechtsidentität beruht laut Butler eben nicht auf biologischer Differenz, sondern auf Performanzen - wiederholten, praktizierten Diskursen bzw. Akten. Kulturelle Identität geht nicht in nationalstaatlichen oder ausschließlich ethnischen Grenzen auf, sondern wird etwa bei Bhabha als praktizierte, performative Wiederholung, als Inszenierung von Identität auf der Grundlage von Begehren und Kopieren von Eigenem und Fremden gefasst (vgl. Bhabha 2001; vgl. auch Hall 1999). Und auch die Identität eines Dings wird - folgt man Latour - über Performanzen hergestellt. ${ }^{14}$ Dabei entsteht in der Performanz ein Bedeutungsüberschuss, weshalb man sie mit Betram (2003) auch als so genannte Praxis-Überschuss-Theorien bezeichnen kann. ${ }^{15}$ Allerdings wird dieser Bedeutungsüberschuss innerhalb der studies unterschiedlich bewertet: Wo Latour eher die kontingente Seite jenes Bedeutungsüberschusses, etwa bei Louis Pasteurs Entdeckung des Milchsäureferments (vgl. Latour 2000: 137-210) betont, stellen Bhabha und Butler das subversive Potential in den Mittelpunkt. Das liegt vor allem daran, dass sich sowohl Bhabba als auch Butler vorwiegend auf (kulturelle und sexuelle) Minderheitsidentitäten konzentrieren, die massiven Vereindeutigungszumutungen durch dominante Gruppen ausgesetzt sind.

Zusammenfassend lässt sich sagen, dass die Theoretisierung des Subjekts trotz der unterschiedlichen Begriffe und Kontexte ähnlich ist: Identität ist ein Gewebe von Differenzen, ohne

14) So ist etwa das Ereignis »der Entdeckung/Erfindung/Konstruktion des Milchsäureferments durch Pasteur im Jahre 1857 « (Latour 1996a: 87) durch die performative Assoziation verschiedener heterogener Entitäten entstanden, wie »Pasteur, die naturwissenschaftliche Fakultät von Lille, Liebig, die Käsereien, die Laborausrüstungen, die Bierhefe, den Zucker und schließlich das Ferment« (Latour 1996a: 106).

15) Bertram (2003: 212) sieht zwei Diskussionsstränge, die im Laufe des 20. Jahrhunderts »einen Vorrang [der Praxis, J.R./M.W.] in Fragen der Theorie « thematisiert haben. Einerseits den >Praktizismus etwa von Sellars und Brandom, welcher »Gehalte als durch Praktiken konstituiert « versteht und andererseits >Praxis-Überschuss-Theorien $<$, die den »Praktiken immer einen Überschuss über die sie prägenden beziehungsweise von ihnen geprägten Gehalte unterstellen, wodurch Praxis als Subversion und Veränderung von Gehalten in den Blick kommt (Bertram 2003: 214). 
zentrale Struktur, in ihrer Entwicklung unabgeschlossen und ohne > wahren< Kern. Letzteres zeigt, dass die Dezentrierung des Subjekts mit einer Dekonstruktion - und nicht Destruktion der für das westliche moderne Denken so zentralen Subjekt/Objekt-Trennung einhergeht, denn der Ausgangspunkt ist nicht >das< Subjekt, sondern seine komplexe Verschlungenheit mit anderen menschlichen wie nicht-menschlichen Aktanten. Und auch die Identität eines Aktanten ist >bloß< die eines Quasi-Subjekts oder Quasi-Objekts (vgl. Latour 2002; Serres 1987). Sie sind und bleiben sozio-technische Netze. Somit stellt sich nicht nur der Actor-Network Theory die Frage, »was ereignet sich?« anstatt »wer handelt?« (Gomart/Hennion 1999: 225).

\section{Politik und Kritik der Moderne}

Auf Grundlage dieses Performanzmodells kommen postcolonial, gender und science studies zu einer Neukonzipierung im Sinne einer Entgrenzung des Politischen. Politik bzw. Macht wird als in Anlehnung an Michel Foucaults (1994) >Mikrophysik der Macht< als Mikromacht in subtilen Praxiszusammenhängen verortet - ob in medialen Diskurspraktiken, politischen Repräsentationspraktiken, Sprech- und Bezeichnungspraktiken oder wissenschaftlichen Visualisierungspraktiken. Damit dehnen sie den Bereich des Politischen massiv auf solche Sphären des Sozialen aus, die bislang eher als Schauplätze machtfreier Begegnungsstätten galten.

Allerdings sind durchaus unterschiedliche politische Strategien festzumachen. Während Bhabha (2000) und Butler (1995) eine »Politik des Performativen« entwickeln, entwirft Latour (2001) die Vision eines »Parlament der Dinge«. Im Gegensatz zu Bhabhas und Butlers sehr gezielter Subversion der Diskurse der Normalität, scheint es Latour eher um eine grundsätzliche Aufdeckung der Netze und Performanzen zu gehen. Auch ihr damit verbundenes ureigenstes (forschungs-)politisches Selbstverständnis ist ein anderes: Während Butler, Bhabha und auch Haraway sich selbst als politische Agenten verstehen, scheint Latour durchaus distanzierter. Nichtsdestotrotz stimmen sie darin überein, dass das Politische auf kein Außen mehr verweist: »in der Politik gibt es keine Transzendenz«(Latour 1997: 50). Somit ist eben nicht wirklich >das Politische<, sondern das >Politik machen< von Interesse. Deswegen spielen für sie strategische Allianzen und Dialog (auch in Differenzen) eine wichtige Rolle in neuen Formen von Politik. Eng damit verbunden ist die Suche nach Widerstand und die Entfaltung von neuen Modellen der Identitätspolitik, die sich gezielt gegen >einfache< Emanzipationslogiken positionieren. Anstelle > großer Utopien< wird für ein lokales und am konkreten Problem orientiertes Engagement plädiert, ohne den Widerstand auf die Ebene einzelner Akteure zu fragmentieren. Statt > von außen< auf die Machtverhältnisse einzuwirken, werden Strategien der Subversion >innerhalb< der naturalisierten und verdinglichten Denk-, Wahrnehmungs- und Handlungsschemata ausgelotet.

Gemeinsam ist den postcolonial, gender und science studies eine starke Kritik an >der Moderne als imperialistische Teleologie des Westens. Gewissermaßen wird die >Erfolgsgeschichte der Moderne vom exterritorialen Standpunkt des Anderen, des von der Moderne ausgrenzten Fremden, umgeschrieben und in Frage gestellt. ${ }^{16}$ In diesem Sinne stehen sie in der Tradition Foucaults, dessen historische Studien zur modernen (wissenschaftlichen) Denkpraxis weniger an den Erfolgen denn an den Verdrängungen der Moderne ansetzen.

So wenden sich etwa postkoloniale TheoretikerInnen in ihrer Positionierung auf Seiten der schmerzlichen Erfahrung von MigrantInnen der >dritten Welt< überdeutlich gegen einen latenten (eurozentrischen) Objektivismus moderner Kulturtheorien. Dieser messe die Geschichten und sozialen Formationen aller Gesellschaften an einer für das westliche Europa

16) Vgl. hierzu exemplarisch Sudhir Chandras (2004) Kritik am »Siegeszug des imperialistischen Modernisierungsdiskurses « aus der Sicht der indischen Gesellschaft oder Chakrabartys (2000) programmatische Losung »Provincializing Europe $\ll$. 
und das nördliche Amerika typischen historischen Entwicklung, so dass die Unterschiede und Besonderheiten nichtwestlicher Gesellschaften und Menschen(-gruppen) dementsprechend in einer >Sprache des Mangels $<$ beschrieben würden (vgl. Conrad/Randeria 2002: 12). Aktuelle Geschlechterstudien kritisieren den latenten (patriarchalen) Objektivismus moderner Geschlechtertheorien durch den Verweis auf >Leidens ‘geschichten sexueller Minoritäten, während die science studies den wissenschaftlich-technischen Objektivismus der Moderne anhand ihrer selbsterzeugten ökologischen wie technologischen Folgeschäden (Atomkraft, Ozonloch, BSE usw.) kritisieren.

Im gewissen Sinne machen sie auf drei marginalisierte Andere der Modernisierung und der Kultur- und Sozialwissenschaften aufmerksam: Dem westlichen Mythos von der zivilisierten und fortschrittlichen Moderne wird in den postcolonial studies sein >barbarischer Spiegel des Kolonialismus und Imperialismus vorgehalten. Modernisierung - und neuerdings auch Globalisierung - werden konsequent aus der Perspektive des Fremden, der Peripherie, d.h. den ehemals (sic!) kolonialisierten Ländern und marginalisierten ethnischen Minderheiten (v.a. in den Metropolen der ehemaligen Kolonialmächte) her gedacht. ${ }^{17}$ Zentral sind dabei die ambivalenten ökonomischen, kulturellen und politischen Machtrelationen zwischen, aber v.a. auch innerhalb von Nationen, Kulturen und Rassen, die ihre Wurzeln in der Kolonialgeschichte haben und im »the on-going colonial present« (Bhabha) der Globalisierung nachwirken (vgl. Moore-Gilbert 1997: 12).

Eine Kritik der Moderne im Kontext der gender studies bedeutet v.a. die Kritik einer modernen >Diktatur der Heterosexualität< und maskulinen Deutungsmacht der Geschlechterdifferenz. Butler (1991: 19) zieht die These vom >universalen Patriarchat< des Feminismus in Zweifel und verschiebt den Blick auf die Herrschaft der binären Geschlechtercodierung und »Zwangsheterosexualität« (vgl. Butler 1991: 9). In ihren Stigmatisierungsstudien Homo-, Trans-, und Intersexueller macht sie auf die reale Gewalt der Diskurse der Zweigeschlechtlichkeit und Heterosexualität als natürliche Norm aufmerksam. Ziel ihres Gender Trouble ist die Subversion dieser Diskurse.

Wie Butler geht es auch Latour $(2000 ; 2001 ; 2002)$ in >seinen< science studies um die Infragestellung der Moderne als Dualismus von Natur und Kultur. Sein Hauptkritikpunkt ist die Konzipierung der Moderne als ein Projekt wissenschaftlich-technischer Rationalität, das zwischen politischer und epistemologischer Repräsentation, zwischen Kultur und Natur strikt trennt, gemäß der Vorstellung von Kultur als Ergebnis immanenter zeitlich und räumlich gebundener Verhandlungen und Konstruktionsleistungen und Natur als transzendentem Bereich von Universalität, Fakten und Wahrheit. Paradoxerweise hat aber seiner Ansicht nach genau diese strikt dualistische »Verfassung der Moderne« die Ausbreitung der Hybriden gestärkt: Sie konnten sich vermehren, gerade weil sie nicht denkbar waren, weil sie weder der Natur noch der Gesellschaft zugeschlagen werden konnten (vgl. Latour 2002: 81). Selbst in der heutigen Zeit, in der die Hybriden in Form von Gentechnologien, Ozonloch oder Prionen allgegenwärtig und mächtig geworden sind, fehlt es uns nach Latour an Einsicht, dass »wir nie modern gewesen sind «. Auch Butler und Bhabha gehen von einer Vermehrung wie gestiegenen Sichtbarkeit von Hybriden in der heutigen Zeit aus. ${ }^{18}$ Sie alle insistieren aber gleichzeitig darauf, dass die grenzüberschreitenden Phänomene - weder im Hinblick auf Kulturen noch auf Geschlechter oder Technik wirklich >neu< sind (vgl. Schröter 2002, Latour 2002). Es ist ihr Stellenwert innerhalb der Forschung und Gesellschaft, welcher als >neu< bewertet wird und werden soll (vgl. Nassehi 1999).

17) Oder wie Costa (2005: 286) in seiner Synopse argumentiert: »Die postkoloniale Reinterpretation moderner Geschichte versucht, den Kolonisierten in die Moderne einzufügen - nicht als Gegenpart zum Westen oder als Synonym der Rückständigkeit, des Traditionellen, des Mangels, sondern als konstitutiven und essenziellen Bestandteil dessen, was man als Moderne diskursiv konstruiert hat.« 
Insgesamt zeigt die systematische Zusammenschau, dass zwischen den in der deutschen Soziologie allzu häufig isoliert betrachteten postcolonial, gender und science studies theoretische Konvergenzen existieren, die trotz des unterschiedlichen Phänomenbereichs in einem differenztheoretischen und performativen Identitätskonzept und einer kritischen Neubestimmung eines politischen >Projekts der Moderne< münden:

Tabelle 1: tabellarischer Vergleich der postcolonial, gender und science studies

\begin{tabular}{|c|c|c|c|}
\hline & Postcolonial Studies & Gender Studies & Science Studies/ ANT \\
\hline $\begin{array}{l}\text { Philosophische } \\
\text { Quellen/Wurzeln }\end{array}$ & $\begin{array}{l}\text { Derrida } \\
\text { Fanon } \\
\text { Foucault } \\
\text { Lacan }\end{array}$ & $\begin{array}{l}\text { Derrida } \\
\text { Foucault } \\
\text { Lacan }\end{array}$ & $\begin{array}{l}\text { Deleuze } \\
\text { Foucault } \\
\text { Serres }\end{array}$ \\
\hline $\begin{array}{l}\text { Zwischenräume/ } \\
\text { Bekämpfter } \\
\text { Dualismus }\end{array}$ & $\begin{array}{l}\text { Eigene und fremde } \\
\text { Kultur } \\
\text { global und lokal }\end{array}$ & $\begin{array}{l}\text { männlich und weiblich } \\
\text { sex und gender }\end{array}$ & $\begin{array}{l}\text { Natur und Kultur } \\
\text { Technik und } \\
\text { Gesellschaft }\end{array}$ \\
\hline $\begin{array}{l}\text { Hybride/Untersu- } \\
\text { chungsphänomene }\end{array}$ & $\begin{array}{l}\text { postkoloniale } \\
\text { MigrantInnen }\end{array}$ & $\begin{array}{l}\text { Transsexuelle, } \\
\text { Intersexuelle }\end{array}$ & $\begin{array}{l}\text { Cyborg, Ozonloch, } \\
\text { BSE }\end{array}$ \\
\hline $\begin{array}{l}\text { Identität } \\
\text { Performanz }\end{array}$ & $\begin{array}{l}\text { (Kultur-)Identität als } \\
\text { diskursive Positio- } \\
\text { nierung }\end{array}$ & $\begin{array}{l}\text { (Geschlechts-)Identität } \\
\text { als performativer Akt }\end{array}$ & $\begin{array}{l}\text { (Objekt-)Identität als } \\
\text { Netzwerk(-effekt) }\end{array}$ \\
\hline Politik & $\begin{array}{l}\text { Strategic Essentialism } \\
\text { Mimikry }\end{array}$ & $\begin{array}{l}\text { Politik des } \\
\text { Performativen }\end{array}$ & Parlament der Dinge \\
\hline Moderne & $\begin{array}{l}\text { Kolonialismus und } \\
\text { Imperialismus }\end{array}$ & $\begin{array}{l}\text { patriarchale Herrschaft } \\
\text { Zwangsheterosexuali- } \\
\text { tät } \\
\text { Zweigeschlechtigkeit }\end{array}$ & $\begin{array}{l}\text { wissenschaftlich- } \\
\text { technische Rationalität }\end{array}$ \\
\hline
\end{tabular}

\section{Rekonfiguration des Sozialen? Postcolonial, gender und science studies als Heraus- forderung der Soziologie}

Durch die Verwerfung jeglichen linearen Fortschrittsgedankens können postcolonial, gender und science studies in einem umfassenderen Sinn auch als Herausforderung der mainstreamSoziologie betrachtet werden, die aus dem »Geist der Moderne« entstanden ist sowie ihn durch ihre Modernisierungstheorien entscheidend mitgeprägt hat. Dennoch stellt sich hier die Frage, ob diese Herausforderung lediglich in einer - wenn auch umfassenden - Kritik der Moderne mündet. Damit wären die studies nicht wirklich >innovativ<, schließlich ist der theoretische Reflex auf eine allzu >einfache< Modernisierungsdiskussion keineswegs neu (vgl. Foucault 1994; Beck/Giddens/Lash 1997). Die Frage ist also, ob die studies nicht nur >alte zentristische Identitätskonzepte, binaristische Differenzmodelle und positivistische Wissen-

18) Einher mit ihrer Kritik an der Moderne geht die Problematisierung der wissenschaftlichen Repräsentation. Die postcolonial studies machen auf die Missrepräsentation des Fremden in der westlichen Ethnologie, Literatur und Geschichtswissenschaft aufmerksam (Bhabha 2000; Said 1978; Spivak/ Guha 1988; Conrad/Randeria 2002), genauso wie die der postkolonialen Minderheiten in den globalen Metropolen (Hall 1994; 1999; Gilroy 1993). Butler (1991; 1995) macht auf die Missrepräsentation der Geschlechtsidentität durch die binäre männlich/weiblich- und sex/gender-Brille aufmerksam. Und schließlich empfiehlt Latour, an den WissenschaftlerInnen als Sprecher der nichtmenschlichen Wesen und »ihrem Vermögen, im Namen ihrer Mandaten zu sprechen, stark, aber nicht definitiv [zu] zweifeln« (Latour 2001: 95). 
schaftsverständisse mit ihren Grenzgestalten in Frage stellen können, sondern ob sie auch jenseits von de-konstruktivistischen >Einzelfallstudien< neue sozialtheoretische Angebote im Hinblick auf Identitäts-, Differenz- und Integrationskonzepte machen, die ihre dauerhafte intra- und intersystemische Konstituierung und Perpetuierung unterstreichen.

Zumindest im Hinblick auf das Akteurs- und Handlungskonzept finden sich - wenn auch mit unterschiedlichen Schwerpunktsetzungen - sowohl in postcolonial als auch in gender und science studies fruchtbare Rekonfigurationen des Sozialen: So schlägt etwa Latour anstelle des >endogenen< bzw. >(ethno-)zentrischen< soziologischen Subjektbegriffs den semiotischen Begriff des Aktanten vor. Ein Aktant ist »something that acts or to which activity is granted by others « (Latour 1996b: 373). Das Besondere an Aktanten ist, dass sie als menschliche und nicht-menschliche Mischwesen heterogene Netze knüpfen. Sie leisten Vermittlungs- oder Übersetzungsarbeit. ${ }^{19}$ Anstatt Handeln als intentionales Handeln reflexiver bzw. rationaler (Einzel-)Akteure zu begreifen und damit Gefahr zu laufen, zentrale Prämissen des teleologischen Modernitätsverständnisses zu reproduzieren - nämlich einer bestimmten modernen Subjektivitätsform - stellt sich Handlung als Übersetzung dar, d.h. Handlungsziele von menschlichen Akteuren werden umdefiniert und durch die Assoziation mit nichtmenschlichen Entitäten verschoben. Aber auch die Funktionen der nicht-menschlichen Entitäten werden durch die menschlichen Akteure umdefiniert und verschoben. ${ }^{20}$ Demnach ist $>$ Handeln< die Fähigkeit, die aus der Verbindung der Aktanten als Netzwerk entsteht.

Auch Butler verzichtet auf den klassisch soziologischen Begriff der Handlung und ersetzt ihn ebenfalls durch einen sprachwissenschaftlichen Ausdruck: den des performativen Akts. Performative Akte beschreiben eine ritualisierte Inszenierung gesellschaftlich bereits eingeführter Bedeutungen. Es sind soziale, höchst regulierte Praktiken, die eine ständige Wiederholung von Normen erzwingen (vgl. Butler 1995: 22). Neben der Abgrenzung zum Begriff des Expressiven richtet sich Butler damit gleichermaßen gegen eine zu individualistische Lesart. Nicht das Subjekt tritt in Aktion; es muss seinen Subjektstatus erst durch die performativen Akte erlangen (vgl. Reuter 2004). Wenn auch über unterschiedliche Wege - über Austin im Falle Butlers, über Greimas im Falle Latours - so kommen beide zu dem Schluss, dass Handlungsfähigkeit wie auch Identität immer (wieder) hergestellt werden muss.

19) Dabei versteht Latour unter einer Vermittlung keine Übermittlung im Sinne des klassischen Sender/ Empfänger-Modells. Genauso ist eine Übersetzung nie eine 1:1-Übertragung zweier von einander unabhängig bestehender Sprachen, sondern »eine Verschiebung, Drift, Vermittlung und Erfindung, es ist die Schöpfung einer Verbindung, die vorher nicht da war und die beiden ursprünglichen Elemente oder Agenten in bestimmtem Maße modifiziert (Latour 2000:217f). Übersetzung beinhaltet eben traductore und traditore - übersetzen und betrügen (vgl. Law 1999: 1 und Clifford 1997: 39; vgl. hier auch die Metapher der Übersetzung in den postcolonial studies etwa bei Bhabha 2000 oder Niranjana 1992).

20) Eine beliebige Handlung, meist ganz klassisch im Sinne von menschlich und intentional, gerät ins Stocken. Damit das Ziel dennoch erreicht wird, wird das Handlungsprogramm an ein Objekt delegiert. Dieser Übersetzungsprozess ist letztlich einer von Zeichen in Materie. So wird etwa die Anweisung »an der Schule langsam vorbei fahren« in den Beton der »schlafenden Gendarmen« gegossen, so dass die Rückübersetzung nun primär wohl eher »Schonen Sie ihre Stoßdämpfer « lautet (vgl. Latour 1996a: 9). Dabei ist eine Handlung nicht auf eine Entität, ob menschlich oder nicht-menschlich, reduzierbar. Sie ist zusammengesetzt und verteilt. »Handeln« ist ein Vermögen eines ganzen Ensembles von Aktanten (vgl. Latour 2000: 221). So lässt sich etwa der Gebrauch von Schusswaffen weder auf den »freien Willen «' des Akteurs noch auf die »Macht« oder »Funktion« der Waffe herunterbrechen. Es ist nicht die Waffe, die den Menschen zum Mörder und auch nicht allein der Mensch, der die Waffe zum Tötungsinstrument macht. Handeln tut der Hybrid oder Aktant »Waffen-Bürger« oder »Bürger-Waffe« (vgl. Latour 2000: 213-219). Für eine Kritik an Latours `Empirie< als auch seiner semiotischen Argumentation innerhalb der science studies, die nichtsdestotrotz materielle Handlungsfähigkeit anerkennt vgl. Rammert/Schulz-Schaeffer (2002) und Pickering (1993). 
Auch im Feld der postcolonial studies, vor allem in den jüngeren, empirisch ausgerichteten Arbeiten wird durch den taktischen (Wieder-)Einsatz des Praxisbegriffs ein modifiziertes Verständnis des Handelns, des Akteurs, des Sozialen schlechthin zum Ausdruck gebracht (vgl. Reckwitz 2003). Schließlich dient ihr Credo »Kultur ist (translokale) Praxis« nicht nur der Abgrenzung von einem latenten (eurozentrischen) Objektivismus moderner Kulturtheorien. Der Praxisbegriff markiert darüber hinaus, dass sich auch das empirische Handeln genauso wenig aus den >mächtigen $<$ gesellschaftlichen (Ungleichheits-)Strukturen wie aus dem personalen Komplex von Motiven und Absichten des rationalen Subjekts ableiten lässt. Ebenso wie die science und gender studies betonen, werden die Aktivitäten von Gesellschaftsmitgliedern (und dies schließt auch nicht-menschliche Mitglieder/Artefakte mit ein) als ein Tun betrachtet, das Dinge geschehen macht oder geschehen lässt. ${ }^{21}$

Obwohl die studies Angebote für das Neudenken sozialtheoretischer Grundkonzepte von Subjekt und Handlung machen, stellt sich die tendenzielle Auflösung des Subjekts für weiterreichende handlungstheoretische Anschlüsse als problematisch dar. Schließlich wird in allen drei studies Subjektivität nicht als Ursprung, sondern als ein Effekt diskursiver und sozialer Praktiken betrachtet. Diese Problematisierung des sprechenden und handelnden Subjekts raubt den sonst so verfeindeten Handlungstheorien (interpretativ vs. intentional) ihre gemeinsame Grundlage: das autonome, selbstbestimmte und kohärente Subjekt.

Durch die Betonung von Diskursen, Kontexten oder (Relations-)Netzen entziehen sie sich einerseits der handlungstheoretischen Ebene, aber andererseits kann man sie auch nicht systemtheoretischen, makrosoziologischen oder struktur- und ordnungsorientierten Ansätzen zurechnen. Hiergegen spricht ihre Fokussierung auf Singularität, Differenz und Mikrostrukturen. Ihr großes Interesse besteht gerade darin, das Scheitern oder die desintegrativen, gewaltvollen Aspekte sozialer Ordnung zu thematisieren. Sie zeichnen eher - durchaus auch im Sinne von Laclau/Mouffe (1991) - die »Unmöglichkeit von Gesellschaft« auf. Die Herausforderung für die Gesellschaftstheorie besteht darin, die Dekonstruktion des Subjekts nicht wieder in geschlossene und deterministische Strukturen aufzulösen, wie es strenge Handlungstheoretiker den poststrukturalistisch informierten studies immer wieder vorwerfen. Allerdings stellen sie ein für die Soziologie schwer verdauliches Programm dar, denn gerade die zentralen Fragen der Soziologie nach sozialem Handeln, sozialer Ordnung und sozialen Wandel werden von ihnen auf den Kopf gestellt (vgl. zu diesem Verständnis von Sozialtheorie Joas/Knöbl 2004). Was es der >klassischen< Soziologie so schwierig macht ist, dass es für sie keine Akteure und auch keine Struktur gibt. Es gibt keinen Akteur, der intentional vielleicht noch durch die Situation eingeschränkt handelt. Aber es gibt auch nicht die Struktur, ob die individuelle, d.h. kognitiv oder psychisch, oder die soziale (Klassen, Milieus ...) oder die kulturelle, die den Akteur in seinem Handeln bestimmt. Sie kommen sowohl ohne >das $<$ Subjekt als auch ohne $>$ die $<$ Gesellschaft aus. ${ }^{22}$ Kontingenz und Performativität verweisen auf ein nicht-subjektivistisches Verständnis sozialer Praxis, das auf den Rückgriff auf einen holistischen und geschlossenen Rahmen >der < Gesellschaft verzichtet. So sind sie parasitäre oder spektrale Soziologien, die »...in den Zwischenräumen der Soziologie...spuken. [und]... sich als das Un-Heimliche der Soziologie, als Sinnbruch in der Soziologie, ohne sich außerhalb der Soziologie zu positionieren [, bestimmen]« (Stäheli 2000: 72).

21) Vgl. zum Handlungsbegriff der science studies gut: Hirschauer (2004).

22) Obwohl Butler ihr Konzept des performativen Aktes an ein gesellschaftlich reguliertes Normen- und Sanktionssystem rückbindet und damit die Vorstellung >der < Gesellschaft - zumindest stellenweise - aufrecht erhält. 


\section{Fazit}

Auch wenn man ihrem >radikalen< sozialtheoretischen Programm nicht folgen will, so muss sich die Soziologie zu einem gewissen Grad eingestehen, dass postcolonial, gender und science studies auf die Diskontinuitäten und Brüche von Modernisierung aufmerksam machen, die in einer >ordentlichen<, weil eindeutig-klassifizierenden Sichtweise bzw. in einem »generalisierenden Theoriestil« (Costa 2005: 292) häufig aus dem Blick geraten ${ }^{23}$ : angefangen von den Kontingenzen der Individualisierung sexueller Orientierungen und Identitäten über Re-Lokalisierung von globalen Akteuren bis hin zur Ökonomisierung der Wissenschaften.

Besonders in zweierlei Hinsicht sind die poststrukturalistisch informierten studies soziologisch interessant und produktiv. Zum einen ist es ihr zeitdiagnostisches Potential, welches sie für die Soziologie attraktiv macht: Individualisierung, (Ent-)Differenzierung, Technisierung, Globalisierung u.ä. sind für sie nicht nur die Folie, auf der sie bestimmte Phänomene diskutieren, sondern jene Prozesse, die durch die Phänomene selbst erst konstituiert werden. Zum anderen stellt ihre Kritik an grundlegenden (analytischen) Dualismen und Konzepten wie die Nation, die Kultur, die Wissenschaft, die Technik und die Frauen - an denen auch die Soziologie nicht arm ist - eine Herausforderung dar. Schließlich sind solche Dualismen unterkomplex und können die Vielfalt des gesellschaftlichen Lebens nicht hinreichend erfassen. Doch leider bleibt es oft bei dieser Dekonstruktion von grundlegenden Dualismen. Nicht dass eine (Selbst-)Kritik an der Soziologie und ihrer Konzepte und Begriffe schlecht wäre. Gerade Bhabha, Butler und Latour haben wichtige Debatten angestoßen und somit zu einer Revitalisierung sozialtheoretischer und sozialphilosophischer Fragen innerhalb der (jeweiligen Bindestrich-)Soziologie beigetragen. Aber häufig ist das von den studies angestoBene »Queering Soziologie« - wie Nina Degele (2003: 22f.) in Bezug auf die gender studies einräumt - eine theoretische Irritation, ohne sich im Design empirischer Forschung niederzuschlagen. Gerade die Soziologie behandelt weiterhin Geschlecht und Ethnizität als unabhängige Strukturkategorien, anstatt ihre Objektivität nicht als Ursache, sondern als Ergebnis von Kontexten und Verweisungszusammenhängen zu betrachten. Diese fehlende >Übersetzung < in die Forschungspraxis mag zum einen daran liegen, dass die Kritik der studies durch ihre komplexe und essayistische Sprache, die mythische und narrative Mittel einsetzt, teilweise selbst wie eine >Fremdsprache< erscheint, die einer > Übersetzung < bedarf. ${ }^{24}$

Soziologie und postcolonial, gender und science studies können happy together sein (Degele 2003: 26), wenn sie sich in der Konstruktion ihrer Gegenstände, vor allem aber in der Konstruktion ihrer Art, Wissenschaft zu treiben, aufeinander einlassen. Die kritische Botschaft der studies, den kolonialen, heteronormativen und situierten Blick >westlicher< Wissenschaftler zu >entlarven<, scheint jedenfalls angekommen zu sein. Aber es bleibt der Wunsch nach empirischen Konsequenzen, damit die studies nicht nur als punktuelle Ironie und Parodie der Soziologie wahrgenommen werden, sondern ihre analytischen Konzepte laut Costa (2005: 293) auch einen nachhaltigen Eingang in die Soziologie finden.

23) Martin Fuchs (2004: 440) spricht in diesem Zusammenhang auch vom Ende eines »Denkens in Modellen und Schablonen«. In seinem ausführlichen Plädoyer für eine Umstellung der soziologischen Blickrichtung der Fremdanalyse - vom Vergleich auf die Analyse einer methodischen Interkulturalität - wirbt er gewissermaßen für die Bereitschaft, leitende Theoreme zu korrigieren und sich auf die Besonderheit des Einzelfalls einlassen zu können. Dies schließt das Bewusstsein einer letztendlichen Unhintergehbarkeit des Anderen ebenso ein wie die Konfrontation mit Bruch- und Kontingenzlinien innerhalb der >eigenen< Kultur.

24) Allerdings muss man den studies zu Gute halten, dass es ihnen damit gerade darum geht, durch Sprache die konventionelle Vorstellung von Wissenschaft zu unterlaufen und ihre rhetorische Konstitution offen zu legen. 


\section{Literatur}

Allolio-Näcke, Lars / Kalscheuer, Britta / Manzeschke, Arne (Hrsg., 2005). Differenzen anders denken. Bausteine zu einer Kulturtheorie der Transdifferenz. Frankfurt a. M.: Campus.

Angermüller, Johannes (2004): > French Theory < in den USA. Diskursanalytische Betrachtungen eines internationalen Rezeptionserfolgs, in: Sociologia Internationalis 42, S.71-101.

Bauman, Zygmunt (1995): Moderne und Ambivalenz. Das Ende der Eindeutigkeit. Frankfurt a. M.: Fischer.

Beck, Ulrich / Giddens, Anthony / Lash, Scott (1997): Reflexive Modernisierung. Eine Kontroverse. Frankfurt a. M.: Suhrkamp.

Belliger, Andréa / Krieger, David J. (Hrsg., 2006): ANThology. Ein einführendes Handbuch zur AkteurNetzwerk Theorie. Bielefeld: transcript. (Science Studies).

Bertram, Georg W. (2003): »Im Anfang war die Tat« Praktiken als Basis der Sprache und des Geistes, in: Jens Kertscher / Dieter Mersch (Hrsg.), Performativität und Praxis, München: Fink, S.211-227

Bhabha, Homi ([1993] 2000): Die Verortung der Kultur. Tübingen: Stauffenburg Verlag.

Butler, Judith (1995): Körper von Gewicht. Die diskursiven Grenzen des Geschlechts. Berlin: Berlin Verlag.

Butler, Judith (1991): Das Unbehagen der Geschlechter. Frankfurt a. M.: Suhrkamp.

Callon, Michel (1986): Some Elements of a Sociology of Translation. Domestication of the Scallops and the Fishermen of St. Brieuc Bay, in: John Law (Hrsg.), Power, Action and Belief. A New Sociology of Knowledge? London: Routledge, S.196-233.

Castro Varela, María do Mar/ Dhawan, Nikita (2005): Postkoloniale Teorie. Eine kritische Einführung. Bielefeld: transcript. (Cultural Studies; 12)

Chakrabarty, Dipesh (2000): Provincializing Europe. Postcolonial Thought and Historical Difference. Princeton / Oxford: Princeton University Press.

Chandra, Sudhir (2004): Die repressive Gegenwart, in: Shalini Randeria/ Martin Fuchs/ Antje Linkenbach (Hrsg.), Konfigurationen der Moderne. Sonderheft Soziale Welt. Baden-Baden: Nomos, S. 37-47.

Clifford, James (1997): Routes: Travel and Translation in the late twentieth Century. Cambridge, Mass; London: Havard University Press.

Conrad, Sebastian/ Randeria, Shalini (2002): Einleitung. Geteilte Geschichten - Europa in einer postkolonialen Welt, in: Dies. (Hrsg.), Jenseits des Eurozentrismus. Postkoloniale Perspektiven in den Geschichts- und Kulturwissenschaften. Frankfurt a. M./ New York: Campus.

Costa, Sérgio (2005): Postkoloniale Studien und Soziologie. Differenzen und Konvergenzen, in: Berliner Journal für Soziologie 15, S. 283-294.

Degele, Nina (2003): Happy Together. Soziologie und Gender Studies als paradigmatische Verunsicherungswissenschaften, in: Soziale Welt 54, S. 9-30.

Elliott, Anthony/ Turner, Bryan S. (Hrsg., 2001): Profiles in Contemporary Social Theory. London: Sage.

Foucault, Michel ([1975] 1994): Überwachen und Strafen. Die Geburt des Gefängnisses. Frankfurt a. M.: Suhrkamp.

Friedman, Susan Stanford (2003): Das Sprechen über Grenzen, Hybridität und Performativität. Kulturtheorie und Identität in den Zwischenräumen der Differenz, in: Mittelweg 36 12, S. 34-52.

Fuchs, Martin (2004): Das Ende der Modelle: Interkulturalität statt (Kultur-)Vergleich, in: Shalini Randeria / Martin Fuchs / Antje Linkenbach (Hrsg.), Konfigurationen der Moderne. Sonderheft Soziale Welt. Baden-Baden: Nomos, S. 439-468.

Gilroy, Paul (1993): The Black Atlantic. Modernity and Double Consciousness. London / New York: Verso.

Gomart, Emilie / Hennion, Antoine (1999): A Sociology of Attachment. Music Amateurs, Drug Users, in: John Law / John Hassard (Hrsg.), Actor Network Theory and After. Oxford: Blackwell, S.220-247.

Ha, Kien Nghi (1999): Ethnizität und Migration. Münster: Westfälisches Dampfboot. 
Hall, Stuart (1994): Kulturelle Identität und Diaspora, in: Ders., Rassismus und kulturelle Identität. Ausgewählte Schriften. Hamburg: Argument, S. 26-43.

Hall, Stuart (1999): Kulturelle Identität und Globalisierung, in: Karl H. Hörning / Rainer Winter (Hrsg.), Widerspenstige Kulturen. Cultural Studies als Herausforderung. Frankfurt a. M.: Suhrkamp, S. 393439.

Haraway, Donna (1995): Die Neuerfindung der Natur. Primaten, Cyborgs und Frauen. Frankfurt a. M.: Campus.

Haraway, Donna (1997): Modest_Witness@Second_Millenium. FemaleMan@_Meets_Onco-mouse ${ }^{\mathrm{TM}}$. Feminism and Technoscience. New York / London: Routledge.

Hieber, Lutz/ Villa, Paula-Irene (Hrsg., 2006): Images von Gewicht. Zur sexuellen Identität in der Postmoderne. Bielefeld: transcript. (Gender Studies).

Hirschauer, Stefan (2004): Praktiken und ihre Körper. Über materielle Partizipanden des Tuns, in: Karl H. Hörning / Julia Reuter (Hrsg.), Doing Culture. Neue Positionen zum Verhältnis von Kultur und sozialer Praxis. Bielefeld: transcript.

Joas, Hans / Knöbl, Wolfgang (2004): Sozialtheorie. Zwanzig einführende Vorlesungen. Frankfurt a. M.: Suhrkamp.

Kalscheuer, Britta (2005): Die Widerspenstigkeit von Transdifferenz. In: Peter-Ulrich Merz-Benz / Gerhard Wagner (Hrsg.), Kultur in Zeiten der Globalisierung. Neue Dimensionen einer soziologischen Kategorie. Frankfurt am Main: Humanities Online, S. 69-91.

Keller, Reiner (2004): Diskursforschung. Eine Einführung für SozialwissenschaftlerInnen. Opladen: Leske+Budrich.

Knorr Cetina, Karin (1997): Sociality with Objects: Social Relations in Postsocial Knowledge Societies. In: Theory, Culture \& Society 14, S. 1-30.

Laclau, Ernesto/ Mouffe, Chantal ([1984], 1991): Hegemonie und radikale Demokratie. Zur Dekonstruktion des Marxismus. Wien: Passagen.

Latour, Bruno ([1993] 1996a): Der Berliner Schlüssel. Erkundungen eines Liebhabers der Wissenschaften, Berlin: Akademie Verlag.

Latour, Bruno (1996b): On Actor-network Theory. A few Clarifications, in: Soziale Welt 47, S. 369-382.

Latour, Bruno (1997): Ein neuer Empirismus, ein neuer Realismus. Ein Gespräch mit Gustav Roßler, in: Mittelweg 36 6, S.40-52.

Latour, Bruno (2000): Die Hoffnung der Pandora. Untersuchungen zur Wirklichkeit der Wissenschaft. Frankfurt a. M.: Suhrkamp.

Latour, Bruno (2001): Das Parlament der Dinge. Für eine politische Ökologie. Frankfurt a. M.: Suhrkamp.

Latour, Bruno ([1991] 2002): Wir sind nie modern gewesen. Versuch einer symmetrischen Anthropologie. Frankfurt a. M.: Fischer.

Law, John (1999): After ANT. Complexity, Naming and Topology, in: John Law / John Hassard (Hrsg.), Aktor Network Theory and After. Oxford: Blackwell, S.1-14.

Lemert, Charles (Hrsg., 1999): Social Theory. Multicultural and classic readings. Boulder: Westview.

Lösch, Klaus (2005): Begriff und Phänomen der Transdifferenz. Zur Infragestellung binärer Differenzkonstrukte, in: Lars Allolio-Näcke / Britta Kalscheuer / Arne Manzeschke (Hrsg.), Differenzen anders denken. Bausteine einer Theorie der Transdifferenz. Frankfurt a. M.: Campus, S.26-49.

Moore-Gilbert, Bart J. (1997): Postcolonial Theory. Contexts, Practices, Politics. London / New York: Verso.

Nassehi, Armin (1995): Der Fremde als Vertrauter. Soziologische Beobachtungen zur Konstruktion von Identitäten und Differenzen, in: Kölner Zeitschrift für Soziologie und Sozialpsychologie 47, S.443463.

Nassehi, Armin (1999): Die Paradoxie der Sichtbarkeit. Für eine epistemologische Verunsicherung der (Kultur-)Soziologie, in: Soziale Welt 50, S.349-362. 
Niranjana, Tejaswini (1992): Siting Translation History, Post-Structuralism and the Colonial Context. Berkeley. University of California Press.

Pickering, Andrew (1993): The Mangel of Practice: Agency and Emergence in the Sociology of Science. American Journal of Sociology 99: 559-589.

Rammert, Werner / Schulz-Schaeffer (2002): Technik und Handeln. Wenn soziales Handeln sich auf menschliches Verhalten und technische Abläufe verteilt. In: Dies. (Hrsg.), Können Maschinen handeln? Soziologische Beiträge zum Verhältnis von Mensch und Technik. Frankfurt a. M.: Campus, S. 11-64.

Reckwitz, Andreas (2002): Der soziologische >Kanon<. Disziplinierung oder Grenzüberschreitung?, in: Soziologische Revue 25, S. 247-257.

Reckwitz, Andreas (2003): Grundelemente einer Theorie sozialer Praktiken. Eine sozialtheoretische Perspektive, in: Zeitschrift für Soziologie 32, S. 282-301.

Reuter, Julia (2002): Ordnungen des Anderen. Zum Problem des Eigenen in der Soziologie des Fremden. Bielefeld: transcript.

Reuter, Julia (2004): Körperinszenierungen. Zur Materialität des Performativen bei Judith Butler und Erving Goffman, in: Das Argument 254, S. 105-112

Reuter, Julia / Hörning, Karl. H. (Hrsg., 2004): Doing Culture. Neue Positionen zum Verhältnis von Kultur und sozialer Praxis. Bielefeld: transcript.

Said, Edward (1978): Orientalism. London: Penguin Books.

Schneider, Irmela (2000): Hybridisierung als Signatur der Zeit, in: Caroline Y. Robertson / Carsten Winter (Hrsg.), Kulturwandel und Globalisierung. Baden-Baden: Nomos, S. 175-187.

Schröter, Susanne (2002): FeMale. Grenzverläufe zwischen den Geschlechtern. Frankfurt a. M.: Fischer.

Serres, Michel ([1980] 1987): Der Parasit. Frankfurt a. M.: Suhrkamp.

Spivak, Gayatri Chakravorty (1988): »Can the Subaltern Speak? Speculations on Widow Sacrifice«, in: Cary Nelson / Lawrence Grossberg (Hrsg.), Marxism and the Interpretation of Culture. Chicago: University of Illinois Press, S. 271-313.

Spivak, Gayatri Chakravorty / Guha, Ranajit (Hrsg., 1988): Selected Subaltern Studies. Oxford: Oxford University Press.

Stäheli, Urs (2000): Poststrukturalistische Soziologien. Bielefeld: transcript.

Weber, Jutta (2003): Umkämpfte Bedeutungen. Naturkonzepte im Zeitalter der Technoscience. Frankfurt a. M.: Campus.

Welsch, Wolfgang (1997): Transkulturalität. Zur veränderten Verfassung heutiger Kulturen, in: Irmela Schneider / Christian Werner Thomsen (Hrsg.): Hybridkultur. Medien Netze Künste. Köln: Wienand, S. 67- 90 .

Wieser, Matthias (2004): Inmitten der Dinge. Zum Verhältnis von sozialer Praxis und Artefakten, in: Karl H. Hörning / Julia Reuter (Hrsg.): Doing Culture. Neue Positionen zum Verhältnis von Kultur und sozialer Praxis. Bielefeld: transcript, S. 92-10.

Julia Reuter, FB IV-Soziologie, Universität Trier, Universitätsring 15,

54296 Trier, reuter@uni-trier.de.

Matthias Wieser, Institut für Soziologie, RWTH Aachen, Eilfschornsteinstr. 7, 52062 Aachen, mwieser@soziologie.rwth-aachen.de. 